\title{
2-Cocycles of Original Deformative Schrödinger-Virasoro Algebras 1
}

\author{
Junbo $\mathrm{Li}^{*, \dagger)}$, Yucai $\left.\mathrm{Su}^{\ddagger}\right)$ \\ ${ }^{*)}$ Department of Mathematics, Shanghai Jiao Tong University, Shanghai 200240, China \\ †) Department of Mathematics, Changshu Institute of Technology, Changshu 215500, China \\ ‡) Department of Mathematics, University of Science and Technology of China, Hefei 230026, China \\ E-mail: sd_junbo@163.com,ycsu@ustc.edu.cn \begin{abstract}
introduced and investigated in a series of papers by Henkel, Roger and Unterberger. In the present paper we aim to determine the 2-cocycles of original deformative Schrödinger-Virasoro algebras.
\end{abstract} \\ Abstract. Both original and twisted Schrödinger-Virasoro algebras also their deformations were
}

Key words: Original Schrödinger-Virasoro algebras, 2-cocycles.

\section{§1. Introduction}

Both original and twisted Schrödinger-Virasoro algebras also their deformations were introduced in [3, 4, 5, 11], in the context of non-equilibrium statistical physics, closely related to both Schrödinger Lie algebras and the Virasoro Lie algebra, which are known to be important in many areas of mathematics and physics (e.g., statistical physics). Their vertex representations were constructed in [17]. Later the derivation algebra and automorphism group of the twisted sector were determined in [6]. Almost at the same time, the derivations, central extensions and automorphism group of the extended sector were investigated in 2]. Furthermore, irreducible modules with finite-dimensional weight spaces and indecomposable modules over both original and twisted sectors were considered in [7], and the second cohomology group of a class of twisted deformative sectors was determined in [8] by the authors.

The infinite-dimensional Lie algebras $\mathcal{L}_{\lambda, \mu}(\lambda, \mu \in \mathbb{C})$ considered in this paper called original deformative Schrödinger-Virasoro Lie algebras ( see [11]), possess the same $\mathbb{C}$-basis

$$
\left\{L_{n}, M_{n}, Y_{p} \mid n \in \mathbb{Z}, p \in \frac{1}{2}+\mathbb{Z}\right\}
$$

with the following Lie brackets:

$$
\begin{aligned}
& {\left[L_{n}, L_{m}\right]=(m-n) L_{m+n}} \\
& {\left[L_{n}, Y_{m}\right]=\left(m-\frac{(\lambda+1) n}{2}+\mu\right) Y_{m+n}, \quad\left[Y_{n}, Y_{m}\right]=(m-n) M_{m+n},} \\
& {\left[L_{n}, M_{m}\right]=(m-\lambda n+2 \mu) M_{m+n}, \quad\left[Y_{n}, M_{m}\right]=\left[M_{n}, M_{m}\right]=0 \text {. }}
\end{aligned}
$$

The purpose of this paper is to determine the 2-cocycles of the original deformative Schrödinger-Virasoro algebras $\mathcal{L}_{\lambda, \mu}(\lambda, \mu \in \mathbb{C})$ defined above. The 2-cocycles on Lie algebras

\footnotetext{
${ }^{1}$ Supported by NSF grants 10471091, 10671027 of China, "One Hundred Talents Program" from University of Science and Technology of China.

Corresponding E-mail: sd_junbo@163.com
} 
play important roles in the central extensions of Lie algebras, which can be used to construct many infinite-dimensional Lie algebras, and further to describe the structures and some of the representations of these type Lie algebras. As the cohomology groups are closely related to the structure of Lie algebras, the computation of cohomology groups seems to be important and interesting as well. Maybe due to the reasons stated above, there appeared a number of papers on 2-cocycles and cohomology groups of infinite-dimensional Lie algebras and conformal algebras ( see [1, 2], [9, 10] and [12]-[16]). Now let's formulate our main results below.

Recall that a 2-cocycle on some $\mathcal{L}_{\lambda, \mu}$ is a $\mathbb{C}$-bilinear function $\psi: \mathcal{L}_{\lambda, \mu} \times \mathcal{L}_{\lambda, \mu} \longrightarrow \mathbb{C}$ satisfying the following conditions:

$$
\begin{aligned}
& \psi\left(v_{1}, v_{2}\right)=-\psi\left(v_{2}, v_{1}\right) \quad(\text { skew-symmetry }), \\
& \psi\left(\left[v_{1}, v_{2}\right], v_{3}\right)+\psi\left(\left[v_{2}, v_{3}\right], v_{1}\right)+\psi\left(\left[v_{3}, v_{1}\right], v_{2}\right)=0 \quad \text { (Jacobian identity), }
\end{aligned}
$$

for $v_{1}, v_{2}, v_{3} \in \mathcal{L}_{\lambda, \mu}$. Denote the vector space of 2 -cocycles on $\mathcal{L}_{\lambda, \mu}$ by $\mathcal{C}^{2}\left(\mathcal{L}_{\lambda, \mu}, \mathbb{C}\right)$. For any $\mathbb{C}$-linear function $f: \mathcal{L}_{\lambda, \mu} \longrightarrow \mathbb{C}$, define a 2-cocycle $\psi_{f}$ as follows

$$
\psi_{f}\left(v_{1}, v_{2}\right)=f\left(\left[v_{1}, v_{2}\right]\right), \quad \forall v_{1}, v_{2} \in \mathcal{L}_{\lambda, \mu}
$$

which is usually called a 2-coboundary or a trivial 2-cocycle on $\mathcal{L}_{\lambda, \mu}$. Denote the vector space of 2-coboundaries on $\mathcal{L}_{\lambda, \mu}$ by $\mathcal{B}^{2}\left(\mathcal{L}_{\lambda, \mu}, \mathbb{C}\right)$. A 2 -cocycle $\varphi$ is said to be equivalent to a 2-cocycle $\psi$ if $\varphi-\psi$ is trivial. For a 2-cocycle $\psi$, we denote the equivalent class of $\psi$ by $[\psi]$. The quotient space $\mathcal{H}^{2}\left(\mathcal{L}_{\lambda, \mu}, \mathbb{C}\right)=\mathcal{C}^{2}\left(\mathcal{L}_{\lambda, \mu}, \mathbb{C}\right) / \mathcal{B}^{2}\left(\mathcal{L}_{\lambda, \mu}, \mathbb{C}\right)$ is called the second cohomology group of $\mathcal{L}_{\lambda, \mu}$.

Usually one calls a 2-cocycle $\xi$ on $\mathcal{L}_{\lambda, \mu}$ the Virasoro cocycle, denoted by $\xi_{\text {Vir }}$, if

$$
\xi\left(L_{n}, L_{m}\right)=\frac{n^{3}-n}{12} \delta_{m,-n}, \quad \text { while other components vanishing. }
$$

The main results of the paper can be formulated as follows.

Theorem 1.1. (i) If $\mu \notin\left\{\frac{1}{2} \mathbb{Z}\right\}$, then for any $\lambda \in \mathbb{C}, \mathcal{H}^{2}\left(\mathcal{L}_{\lambda, \mu}, \mathbb{C}\right) \cong \mathbb{C}$ is generated by the Virasoro cocycle.

(ii) For $\mu \in \frac{1}{2}+\mathbb{Z}$ and $\lambda \neq-3,-1,1, \mathcal{H}^{2}\left(\mathcal{L}_{\lambda, \mu}, \mathbb{C}\right) \cong \mathbb{C}$ is generated by the Virasoro cocycle. (iii) For $\mu \in \frac{1}{2}+\mathbb{Z}$ and $\lambda=-3, \mathcal{H}^{2}\left(\mathcal{L}_{-3, \mu}, \mathbb{C}\right) \cong \mathbb{C}^{2}$ is generated by the Virasoro cocycle and an independent cocycle of the form $c\left(L_{n}, Y_{m}\right)=\delta_{n,-m-\mu}$ (all other components vanishing).

(iv) For $\mu \in \frac{1}{2}+\mathbb{Z}$ and $\lambda=-1, \mathcal{H}^{2}\left(\mathcal{L}_{-1, \mu}, \mathbb{C}\right) \cong \mathbb{C}^{3}$ is generated by the Virasoro cocycle and other two independent cocycles $c_{1}$ and $c_{2}$ defined by (all other components vanishing)

$$
c_{1}\left(M_{m}, Y_{n}\right)=\delta_{n,-m-3 \mu}, \quad c_{2}\left(L_{-m}, Y_{n}\right)=\frac{m(m+1)}{2} \delta_{n, m-\mu}
$$


(v) For $\mu \in \frac{1}{2}+\mathbb{Z}$ and $\lambda=1, \mathcal{H}^{2}\left(\mathcal{L}_{1, \mu}, \mathbb{C}\right) \cong \mathbb{C}^{3}$ is generated by the Virasoro cocycle and other two independent cocycles $c_{1}$ and $c_{2}$ defined by (all other components vanishing)

$$
\begin{aligned}
& c_{1}\left(L_{-m}, Y_{n}\right)=m\left(m^{2}-1\right) \delta_{n, m-\mu} \\
& c_{2}\left(L_{-m}, M_{n-2 \mu}\right)=c_{2}\left(Y_{-m-\mu}, Y_{n-\mu}\right)=m\left(m^{2}-1\right) \delta_{m, n}
\end{aligned}
$$

(vi) For $\mu \in \mathbb{Z}$, if $\lambda \neq-1, \mathcal{H}^{2}\left(\mathcal{L}_{\lambda, \mu}, \mathbb{C}\right) \cong \mathbb{C}$ is generated by the Virasoro cocycle; while if $\lambda=-1, \mathcal{H}^{2}\left(\mathcal{L}_{-1, \mu}, \mathbb{C}\right) \cong \mathbb{C}^{2}$ is generated by the Virasoro cocycle and an independent cocycle of the form $c\left(Y_{p}, Y_{q}\right)=-\delta_{q,-p-2 \mu}(p+\mu)$ (all other components vanishing).

Throughout the paper, we denote by $\mathbb{Z}^{*}$ the set of all nonzero integers, $\mathbb{C}^{*}$ the set of all nonzero complex numbers and $\mathbb{C}^{*} \backslash \mathbb{Z}^{*}=\left\{x \mid x \in \mathbb{C}^{*}, x \notin \mathbb{Z}^{*}\right\}$.

\section{$\S 2 . \quad$ Proof the main results}

Let $\psi$ be any 2-cocycle. Define a $\mathbb{C}$-linear function $f: \mathcal{L}_{\lambda, \mu} \rightarrow \mathbb{C}$ as follows

$$
\begin{aligned}
& f\left(L_{n}\right)= \begin{cases}\frac{1}{n} \psi\left(L_{0}, L_{n}\right) \quad \text { if } n \neq 0, \forall \mu \in \mathbb{C}^{*}, \\
\frac{1}{2} \psi\left(L_{-1}, L_{1}\right) \quad \text { if } n=0, \forall \mu \in \mathbb{C}^{*},\end{cases} \\
& f\left(M_{n}\right)= \begin{cases}\frac{1}{n+2 \mu} \psi\left(L_{0}, M_{n}\right) & \text { if } n \neq-2 \mu, \mu \in \frac{1}{2} \mathbb{Z}, \text { or } \mu \notin \frac{1}{2} \mathbb{Z}, \\
\frac{-1}{\lambda+1} \psi\left(L_{1}, M_{-2 \mu-1}\right) & \text { if } n=-2 \mu, \lambda \neq-1, \text { and } \mu \in \frac{1}{2} \mathbb{Z},\end{cases} \\
& f\left(Y_{p}\right)= \begin{cases}\frac{1}{p+\mu} \psi\left(L_{0}, Y_{p}\right) & \text { if } p \neq-\mu, \mu \in \frac{1}{2}+\mathbb{Z}, \text { or } \mu \notin \frac{1}{2}+\mathbb{Z}, \\
\frac{-2}{\lambda+3} \psi\left(L_{1}, Y_{-\mu-1}\right) & \text { if } p=-\mu, \lambda \neq-3 \text { and } \mu \in \frac{1}{2}+\mathbb{Z} .\end{cases}
\end{aligned}
$$

Let $\varphi=\psi-\psi_{f}-\xi_{\text {Vir }}$ where $\psi_{f}$ and $\xi_{\text {Vir }}$ are respectively defined in (1.5) and (1.6), then

$$
\varphi\left(L_{m}, L_{n}\right)=0, \quad \forall m, n \in \mathbb{Z}
$$

The proof of Theorem 1.1 is included in the following three technical lemmas.

Case 1. $\mu \notin \frac{1}{2} \mathbb{Z}$

Lemma 2.1. $\varphi=0$.

Proof. According to (2.2) and (2.3), one has

$$
\varphi\left(L_{0}, Y_{p}\right)=\varphi\left(L_{0}, M_{n}\right)=0, \quad \forall n \in \mathbb{Z}, p \in \frac{1}{2}+\mathbb{Z}
$$

For any $p, q \in \frac{1}{2}+\mathbb{Z}$, using the Jacobian identity on the triple $\left(L_{0}, Y_{p}, Y_{q}\right)$, together with (2.4), we obtain

$$
(p+q+2 \mu) \varphi\left(Y_{p}, Y_{q}\right)=0
$$


which together with our assumption $\mu \notin \frac{1}{2} \mathbb{Z}$ forces

$$
\varphi\left(Y_{p}, Y_{q}\right)=0
$$

For any $m, n \in \mathbb{Z}, p \in \frac{1}{2}+\mathbb{Z}$, using the Jacobian identity on the four triples $\left(L_{m}, Y_{p}, L_{0}\right)$, $\left(L_{m}, M_{n}, L_{0}\right),\left(Y_{p}, M_{n}, L_{0}\right)$ and $\left(M_{m}, M_{n}, L_{0}\right)$ in (1.4) respectively, one has

$$
\begin{aligned}
& (m+p+\mu) \varphi\left(L_{m}, Y_{p}\right)=0, \\
& (m+n+2 \mu) \varphi\left(L_{m}, M_{n}\right)=0, \\
& (p+n+3 \mu) \varphi\left(Y_{p}, M_{n}\right)=0, \\
& (m+n+4 \mu) \varphi\left(M_{m}, M_{n}\right)=0 .
\end{aligned}
$$

Then our assumption $\mu \notin \frac{1}{2} \mathbb{Z}$, together with (2.7) and (2.8) gives

$$
\varphi\left(L_{m}, Y_{p}\right)=\varphi\left(Y_{p}, M_{n}\right)=\varphi\left(L_{m}, M_{n}\right)=0
$$

Similar to the proof of Subcase 1.3 given in [6], one also has

$$
\varphi\left(M_{m}, M_{n}\right)=0, \quad \forall m, n \in \mathbb{Z}
$$

Then this lemma follows.

This lemma in particular proves Theorem 1.1 (i).

Case 2. $\mu \in \frac{1}{2}+\mathbb{Z}$.

Lemma 2.2. For any $\lambda \in \mathbb{C}, \varphi=0$ unless the following subcases:

i) if $\lambda=-1$, then $\varphi\left(M_{m}, Y_{-m-3 \mu}\right)=\varphi\left(M_{0}, Y_{-3 \mu}\right), \varphi\left(L_{-m}, Y_{m-\mu}\right)=\frac{m(m+1)}{2} \varphi\left(L_{-1}, Y_{1-\mu}\right)$;

ii) if $\lambda=-3$, then $\varphi\left(L_{-m}, Y_{m-\mu}\right)=\varphi\left(L_{0}, Y_{-\mu}\right)$;

iii) if $\lambda=1$, then $\varphi\left(L_{-m}, Y_{m-\mu}\right)=m\left(m^{2}-1\right) c_{1}, \varphi\left(Y_{-m-\mu}, Y_{m-\mu}\right)=m\left(m^{2}-1\right) c_{1}^{\prime}$, $\varphi\left(L_{-m}, M_{m-2 \mu}\right)=m\left(m^{2}-1\right) c_{1}^{\prime}$;

for any $m \in \mathbb{Z}$, and some constants $c_{1}, c_{1}^{\prime} \in \mathbb{C}$.

Proof. According to (2.2) and (2.3), one has

$$
\begin{aligned}
& \varphi\left(L_{0}, Y_{p}\right)=0 \text { if } p \neq-\mu, \varphi\left(L_{1}, Y_{-\mu-1}\right)=0 \text { if } \lambda \neq-3 \\
& \varphi\left(L_{0}, M_{n}\right)=0 \text { if } n \neq-2 \mu, \varphi\left(L_{1}, M_{-2 \mu-1}\right)=0 \text { if } \lambda \neq-1 .
\end{aligned}
$$

For any $m, n \in \mathbb{Z}, p, q \in \frac{1}{2}+\mathbb{Z}$, using the Jacobian identity on the triples $\left(L_{0}, Y_{p}, M_{m}\right)$, $\left(L_{0}, M_{m}, M_{n}\right),\left(L_{0}, Y_{p}, Y_{q}\right),\left(L_{0}, L_{m}, M_{n}\right)$ and $\left(L_{0}, L_{m}, Y_{p}\right)$ in (1.4) respectively, together 
with (2.13) and (2.14), one has

$$
\begin{aligned}
& (p+m+3 \mu) \varphi\left(Y_{p}, M_{m}\right)=0, \\
& (m+n+4 \mu) \varphi\left(M_{m}, M_{n}\right)=0, \\
& (p-q) \varphi\left(L_{0}, M_{p+q}\right)+(p+q+2 \mu) \varphi\left(Y_{p}, Y_{q}\right)=0, \\
& (m \lambda-2 \mu-n) \varphi\left(L_{0}, M_{m+n}\right)+(m+n+2 \mu) \varphi\left(L_{m}, M_{n}\right)=0, \\
& (m(1+\lambda)-2(p+\mu)) \varphi\left(L_{0}, Y_{m+p}\right)+2(m+p+\mu) \varphi\left(L_{m}, Y_{p}\right)=0 .
\end{aligned}
$$

For any $m \in \mathbb{Z}, p \in \frac{1}{2}+\mathbb{Z}, p \neq 2 \mu-\frac{m}{2}$, using the Jacobian identity on the triple $\left(Y_{p-4 \mu}, Y_{-p-m}, M_{m}\right)$, one has

$$
(m+2 p-4 \mu) \varphi\left(M_{-m-4 \mu}, M_{m}\right)=0,
$$

which together with (2.16), gives

$$
\varphi\left(M_{m}, M_{n}\right)=0, \quad \forall m, n \in \mathbb{Z}
$$

Using (2.14), the identities (2.17) and (2.18) can be rewritten as

$$
\varphi\left(Y_{p}, Y_{q}\right)=\varphi\left(L_{0}, M_{-2 \mu}\right)=\varphi\left(L_{m}, M_{n}\right)=0 \text { if }(p+q+2 \mu)(m+n+2 \mu) \neq 0 .
$$

The identities (2.13) and (2.19) together give

$$
\varphi\left(L_{m}, Y_{p}\right)=0 \text { if } m+p \neq-\mu, \quad \varphi\left(L_{0}, Y_{-\mu}\right)=0 \quad \text { if } \lambda \neq-3
$$

From (2.4), (2.15) and (2.20) $-(2.22)$, the left components we have to present in this case are listed in the following $\left(\forall n \in \mathbb{Z}, p \in \frac{1}{2}+\mathbb{Z}\right)$ :

$$
\varphi\left(Y_{n}, L_{-\mu-n}\right), \varphi\left(L_{-2 \mu-n}, M_{n}\right), \varphi\left(Y_{-2 \mu-p}, Y_{p}\right) \text { and } \varphi\left(M_{n}, Y_{-n-3 \mu}\right) .
$$

Step 1. The computation of $\varphi\left(M_{n}, Y_{-n-3 \mu}\right), \forall n \in \mathbb{Z}$.

For any $m, n \in \mathbb{Z}$, using the Jacobian identity on the triple $\left(L_{n-m}, Y_{-n-3 \mu}, M_{m}\right)$, one has

$$
\begin{aligned}
& 2(m(1+\lambda)+2 \mu-n \lambda) \varphi\left(M_{n}, Y_{-n-3 \mu}\right) \\
& =(4 \mu+(3+\lambda) n-(\lambda+1) m) \varphi\left(M_{m}, Y_{-m-3 \mu}\right) .
\end{aligned}
$$

If $\lambda=0$, taking $m=0$ in (2.23), one has

$$
\varphi\left(M_{n}, Y_{-n-3 \mu}\right)=\left(1+\frac{3 n}{4 \mu}\right) \varphi\left(M_{0}, Y_{-3 \mu}\right)
$$

Taking (2.24) back to (2.23), one has

$$
(m-n)(m(1+\lambda)+2 \lambda(n+2 \mu)) \varphi\left(M_{0}, Y_{-3 \mu}\right)=0
$$


which forces $\varphi\left(M_{0}, Y_{-3 \mu}\right)=0$ and further $\varphi\left(M_{n}, Y_{-n-3 \mu}\right)=0, \forall n \in \mathbb{Z}$ by (2.24). If $\lambda \neq 0, \frac{2 \mu}{\lambda} \notin \mathbb{Z}$, taking $m=0$ in (2.23), one has

$$
\varphi\left(M_{n}, Y_{-n-3 \mu}\right)=\frac{3 n+4 \mu+n \lambda}{4 \mu-2 n \lambda} \varphi\left(M_{0}, Y_{-3 \mu}\right)
$$

Similarly, one also can prove $\varphi\left(M_{n}, Y_{-n-3 \mu}\right)=\varphi\left(M_{0}, Y_{-3 \mu}\right)=0$.

If $\lambda \neq 0, \frac{2 \mu}{\lambda} \in \mathbb{Z}$, taking $m=0$ in (2.23), one has

$$
\varphi\left(M_{n}, Y_{-n-3 \mu}\right)=\frac{3 n+4 \mu+n \lambda}{4 \mu-2 n \lambda} \varphi\left(M_{0}, Y_{-3 \mu}\right) \quad \text { if } n \neq \frac{2 \mu}{\lambda} .
$$

Taking $(m, n, \lambda)=\left(\frac{2 \mu}{\lambda}, 1,1\right)$ and $(m, n)=\left(\frac{2 \mu}{\lambda}, 0\right)$ in (2.23) respectively, and using (2.25), one has

$$
\varphi\left(M_{\frac{2 \mu}{\lambda}}, Y_{-\frac{2 \mu}{\lambda}-3 \mu}\right)= \begin{cases}\frac{(6 \mu-1)(1+\mu)}{2 \mu-1} \varphi\left(M_{0}, Y_{-3 \mu}\right) & \text { if } \lambda=1, \mu \neq \frac{1}{2}, \\ \varphi\left(M_{1}, Y_{-\frac{5}{2}}\right) & \text { if } \lambda=1, \mu=\frac{1}{2}, \\ \frac{2(1+2 \lambda)}{\lambda-1} \varphi\left(M_{0}, Y_{-3 \mu}\right) & \text { if } \lambda \neq 0,1, \frac{2 \mu}{\lambda} \in \mathbb{Z} .\end{cases}
$$

For the special case $\lambda=1, \mu=\frac{1}{2}$, by taking $n=2$ in (2.25) and $m=2, n=1$ in (2.23), we obtain

$$
\varphi\left(M_{2}, Y_{-\frac{7}{2}}\right)=4 \varphi\left(M_{1}, Y_{-\frac{5}{2}}\right)=-5 \varphi\left(M_{0}, Y_{-\frac{3}{2}}\right)
$$

which gives

$$
\varphi\left(M_{1}, Y_{-\frac{5}{2}}\right)=-\frac{5}{4} \varphi\left(M_{0}, Y_{-\frac{3}{2}}\right) .
$$

Taking $(m, n)=\left(0, \frac{2 \mu}{\lambda}\right)$ in $(2.23)$, one has $\frac{3 \mu(\lambda+1)}{\lambda} \varphi\left(M_{0}, Y_{-3 \mu}\right)=0$, which infers

$$
\varphi\left(M_{0}, Y_{-3 \mu}\right)=0 \quad \text { if } \lambda \neq 0,-1
$$

Then adding up the results obtained after (2.27), one has

$$
\varphi\left(M_{n}, Y_{-n-3 \mu}\right)=\left\{\begin{array}{cl}
0 & \text { if }-1 \neq \lambda \in \mathbb{C}, \mu \in \frac{1}{2}+\mathbb{Z} \\
\varphi\left(M_{0}, Y_{-3 \mu}\right) & \text { if } \lambda=-1, \mu \in \frac{1}{2}+\mathbb{Z}
\end{array}\right.
$$

Step 2. The computation of $\varphi\left(Y_{n}, L_{-\mu-n}\right), \forall n \in \mathbb{Z}$.

Applying the Jacobian identity on the triple $\left(L_{-m}, L_{n}, Y_{m-n-\mu}\right), \forall m, n \in \mathbb{Z}$, one has

$$
\begin{aligned}
& (2 m-n(\lambda+3)) \varphi\left(L_{-m}, Y_{m-\mu}\right) \\
& =(m(\lambda+3)-2 n) \varphi\left(L_{n}, Y_{-n-\mu}\right)+2(m+n) \varphi\left(L_{n-m}, Y_{m-n-\mu}\right) .
\end{aligned}
$$


Replacing $n$ by $-n$ and $m$ by $m+n$ in (2.33) respectively, one has

$$
\begin{aligned}
& (2 m+n(\lambda+3)) \varphi\left(L_{-m}, Y_{m-\mu}\right) \\
& =(m(\lambda+3)+2 n) \varphi\left(L_{-n}, Y_{n-\mu}\right)+2(m-n) \varphi\left(L_{-n-m}, Y_{m+n-\mu}\right) \\
& (2(m+n)-n(\lambda+3)) \varphi\left(L_{-m-n}, Y_{m+n-\mu}\right) \\
& =((m+n)(\lambda+3)-2 n) \varphi\left(L_{n}, Y_{-n-\mu}\right)+2(m+2 n) \varphi\left(L_{-m}, Y_{m-\mu}\right)
\end{aligned}
$$

If $\lambda=-3$, then (2.33) can be rewritten as (by taking $n=1$ )

$$
(m+1) \varphi\left(L_{1-m}, Y_{m-1-\mu}\right)-m \varphi\left(L_{-m}, Y_{m-\mu}\right)=\varphi\left(L_{1}, Y_{-1-\mu}\right)
$$

Using induction on $m$ in (2.33), we obtain

$$
\varphi\left(L_{-m}, Y_{m-\mu}\right)=\varphi\left(L_{0}, Y_{-\mu}\right), \quad \forall m \in \mathbb{Z}
$$

If $\lambda \neq-3$, taking $n=-1$ in both (2.31) and (2.32), together with (2.13), we have

$$
\begin{aligned}
& (2 m-(\lambda+3)) \varphi\left(L_{-m}, Y_{m-\mu}\right)=2(m+1) \varphi\left(L_{1-m}, Y_{m-1-\mu}\right) \\
& (2(m-1)+(\lambda+3)) \varphi\left(L_{1-m}, Y_{m-1-\mu}\right) \\
& =((m-1)(\lambda+3)+2) \varphi\left(L_{-1}, Y_{1-\mu}\right)+2(m-2) \varphi\left(L_{-m}, Y_{m-\mu}\right) .
\end{aligned}
$$

If $(\lambda+5)(\lambda-1) \neq 0$, then identities (2.35) and (2.36) together give

$$
\varphi\left(L_{-m}, Y_{m-\mu}\right)=\frac{2(m+1)((\lambda+1)-m(\lambda+3))}{\lambda^{2}+4 \lambda-5} \varphi\left(L_{-1}, Y_{1-\mu}\right)
$$

Taking $\varphi\left(L_{-m}, Y_{m-\mu}\right)$ and $\varphi\left(L_{1-m}, Y_{m-1-\mu}\right)$ obtained from (2.37) back to (2.35), one has

$$
\frac{m(2 m-\lambda-3)\left(\lambda^{2}+4 \lambda+3\right)}{\lambda^{2}+4 \lambda-5} \varphi\left(L_{-1}, Y_{1-\mu}\right)=0, \quad \forall m \in \mathbb{Z},
$$

which forces ( since the index $m$ can be shifted and our assumption $\lambda \neq-3$ )

$$
\left(\lambda^{2}+4 \lambda+3\right) \varphi\left(L_{-1}, Y_{1-\mu}\right)=0
$$

In another word,

the system consisted of linear equations (2.35) and (2.36) has nonzero solutions if and only if $\lambda=-1$ under our assumption $(\lambda+5)(\lambda-1)(\lambda+3) \neq 0$. (2.39) If $\lambda=-1$, then (2.37) can be rewritten as

$$
\varphi\left(L_{-m}, Y_{m-\mu}\right)=\frac{m(m+1)}{2} \varphi\left(L_{-1}, Y_{1-\mu}\right), \quad \forall m \in \mathbb{Z}
$$


If $\lambda=1$, then (2.35) becomes

$$
(m-2) \varphi\left(L_{-m}, Y_{m-\mu}\right)=(m+1) \varphi\left(L_{1-m}, Y_{m-1-\mu}\right)
$$

which further gives (by taking $m=2$ in (2.41))

$$
\varphi\left(L_{-1}, Y_{1-\mu}\right)=0
$$

Then using induction on $m$ in (2.41), one can deduce

$$
\varphi\left(L_{-m}, Y_{m-\mu}\right)= \begin{cases}m\left(m^{2}-1\right) c_{1} & \text { if } m \geq-2 \mu-1, \\ m\left(m^{2}-1\right) c_{2} & \text { if } m<-2 \mu-1\end{cases}
$$

for some constants $c_{1}, c_{2} \in \mathbb{C}$. One thing left to be done is to investigate the relations between the constants $c_{1}$ and $c_{2}$. If $\lambda=1$, then (2.36) becomes

$$
\begin{aligned}
& (m+2 n) \varphi\left(L_{-m}, Y_{m-\mu}\right) \\
& =(2 m+n) \varphi\left(L_{-n}, Y_{n-\mu}\right)+(m-n) \varphi\left(L_{-n-m}, Y_{m+n-\mu}\right), \\
& (m-n) \varphi\left(L_{-m-n}, Y_{m+n-\mu}\right) \\
& =(2 m+n) \varphi\left(L_{n}, Y_{-n-\mu}\right)+(m+2 n) \varphi\left(L_{-m}, Y_{m-\mu}\right) .
\end{aligned}
$$

which together with each other force

$$
(2 m+n)\left(\varphi\left(L_{-n}, Y_{n-\mu}\right)+\varphi\left(L_{n}, Y_{-n-\mu}\right)\right)=0
$$

Then using (2.43) and (2.44), we obtain $c_{1}=c_{2}$, which together with (2.43) gives

$$
\varphi\left(L_{-m}, Y_{m-\mu}\right)=m\left(m^{2}-1\right) c_{1}, \quad \forall m \in \mathbb{Z}
$$

If $\lambda=-5$, then (2.31) and (2.32) convert to the following form:

$$
\begin{aligned}
& (m-n) \varphi\left(L_{-m}, Y_{m-\mu}\right) \\
& =(n-m) \varphi\left(L_{-n}, Y_{n-\mu}\right)+(m-n) \varphi\left(L_{-n-m}, Y_{m+n-\mu}\right), \\
& (m+2 n) \varphi\left(L_{-m-n}, Y_{m+n-\mu}\right) \\
& =-(m+2 n) \varphi\left(L_{n}, Y_{-n-\mu}\right)+(m+2 n) \varphi\left(L_{-m}, Y_{m-\mu}\right) .
\end{aligned}
$$

Furthermore, taking $n=-1$ in both (2.46) and (2.47), and using (2.13), one has

$$
\begin{aligned}
& (m+1)\left(\varphi\left(L_{-m}, Y_{m-\mu}\right)-\varphi\left(L_{1-m}, Y_{m-1-\mu}\right)\right)=0, \\
& (m-2)\left(\varphi\left(L_{-m}, Y_{m-\mu}\right)-\varphi\left(L_{1-m}, Y_{m-1-\mu}\right)\right)=(m-2) \varphi\left(L_{-1}, Y_{1-\mu}\right) .
\end{aligned}
$$

from which and using (2.13) again, can we deduce the following relation:

$$
\varphi\left(L_{-m}, Y_{m-\mu}\right)=0, \quad \forall m \in \mathbb{Z} .
$$


If $\lambda \notin\{-5,-3,-1,1\}$, the identities (2.37) and (2.38) together also force (2.48) to hold.

Step 3. The computation of $\varphi\left(L_{-2 \mu-n}, M_{n}\right), \forall n \in \mathbb{Z}$.

Using the Jacobian identity on $\left(L_{-2 \mu-m}, L_{n}, M_{m-n}\right), \forall m, n \in \mathbb{Z}$, we obtain

$$
\begin{aligned}
& (m+2 \mu-n(1+\lambda)) \varphi\left(L_{-m-2 \mu}, M_{m}\right) \\
& =((m+2 \mu)(1+\lambda)-n) \varphi\left(L_{n}, M_{-n-2 \mu}\right)+(m+2 \mu+n) \varphi\left(L_{n-m-2 \mu}, M_{m-n}\right) .
\end{aligned}
$$

Replacing $n$ by $-n$ and $m$ by $m+n$ in (2.49), one respectively gets

$$
\begin{aligned}
& (m+2 \mu+n(1+\lambda)) \varphi\left(L_{-m-2 \mu}, M_{m}\right) \\
& =(m+2 \mu-n) \varphi\left(L_{-n-m-2 \mu}, M_{m+n}\right)+((m+2 \mu)(1+\lambda)+n) \varphi\left(L_{-n}, M_{n-2 \mu}\right), \\
& (2 n+m+2 \mu) \varphi\left(L_{-m-2 \mu}, M_{m}\right) \\
& =(m+2 \mu-n \lambda) \varphi\left(L_{-n-m-2 \mu}, M_{m+n}\right)-((n+m+2 \mu)(1+\lambda)-n) \varphi\left(L_{n}, M_{-n-2 \mu}\right) .
\end{aligned}
$$

Then using the same arguments given in Lemma 2.2 of the reference [8], we obtain the following results.

If $\lambda=0$, then

$$
\varphi\left(L_{-m-2 \mu}, M_{m}\right)=\frac{(m+2 \mu)(m+2 \mu+1)}{2} \varphi\left(L_{-1}, M_{1-2 \mu}\right), \quad \forall m \in \mathbb{Z} .
$$

If $\lambda=1$, then

$$
\varphi\left(L_{-m-2 \mu}, M_{m}\right)=(m+2 \mu-1)(m+2 \mu)(m+2 \mu+1) c_{1}^{\prime}, \quad \forall m \in \mathbb{Z} .
$$

for some constant $c_{1}^{\prime} \in \mathbb{C}$.

If $\lambda=-1$, then

$$
\varphi\left(L_{-m-2 \mu}, M_{m}\right)= \begin{cases}(m+2 \mu) \varphi\left(L_{0}, M_{-2 \mu}\right) & \text { if } m \geq-2 \mu \\ (m+2 \mu+2) \varphi\left(L_{0}, M_{-2 \mu}\right) & \text { if } m<-2 \mu .\end{cases}
$$

by (2.21), which can be rewritten as

$$
\varphi\left(L_{-m-2 \mu}, M_{m}\right)=0, \quad \forall m \in \mathbb{Z}
$$

If $\lambda \notin\{-1,0,1\}$, then

$$
\varphi\left(L_{-m-2 \mu}, M_{m}\right)=0, \quad \forall m \in \mathbb{Z}
$$

Step 4. The computation of $\varphi\left(Y_{-2 \mu-p}, Y_{p}\right), \forall p \in \frac{1}{2}+\mathbb{Z}$.

Using the Jacobian identity on $\left(L_{-m}, Y_{-n-\mu}, Y_{m+n-\mu}\right), \forall m, n \in \mathbb{Z}$, one has

$$
\begin{aligned}
& (m(1+\lambda)-2 n) \varphi\left(Y_{-m-n-\mu}, Y_{m+n-\mu}\right) \\
& =2(m+2 n) \varphi\left(L_{-m}, M_{m-2 \mu}\right)-(m(3+\lambda)+2 n) \varphi\left(Y_{-n-\mu}, Y_{n-\mu}\right) .
\end{aligned}
$$


In particular, taking $n=0$ in (2.56), one has

$$
m(1+\lambda) \varphi\left(Y_{-m-\mu}, Y_{m-\mu}\right)=2 m \varphi\left(L_{-m}, M_{m-2 \mu}\right)-m(3+\lambda) \varphi\left(Y_{-\mu}, Y_{-\mu}\right) .
$$

If $\lambda=-1$, then (2.56) becomes

$$
\varphi\left(L_{-m}, M_{m-2 \mu}\right)=\varphi\left(Y_{-\mu}, Y_{-\mu}\right), \quad \forall m \in \mathbb{Z}^{*},
$$

which together with (2.54), forces $\varphi\left(Y_{-\mu}, Y_{-\mu}\right)=\varphi\left(L_{0}, M_{-2 \mu}\right)=0$. Then (2.54) can be rewritten as

$$
\varphi\left(L_{-m-2 \mu}, M_{m}\right)=0, \quad \forall m \in \mathbb{Z}
$$

So in this case, (2.56) can be rewritten as

$$
\varphi\left(Y_{-m-\mu}, Y_{m-\mu}\right)=m \varphi\left(Y_{-1-\mu}, Y_{1-\mu}\right), \quad \forall m \in \mathbb{Z}
$$

Then (2.56) becomes $m(m+n) \varphi\left(Y_{-1-\mu}, Y_{1-\mu}\right)=0$, which implies $\varphi\left(Y_{-1-\mu}, Y_{1-\mu}\right)=0$, and further

$$
\varphi\left(Y_{-m-\mu}, Y_{m-\mu}\right)=0, \quad \forall m \in \mathbb{Z}
$$

If $\lambda=0$, using (2.52) and (2.57), we obtain

$$
\varphi\left(Y_{-m-\mu}, Y_{m-\mu}\right)=m(m+1) \varphi\left(L_{-1}, M_{1-2 \mu}\right)-3 \varphi\left(Y_{-\mu}, Y_{-\mu}\right), \quad \forall m \in \mathbb{Z}^{*}
$$

which together with (2.56), gives $6 \varphi\left(Y_{-\mu}, Y_{-\mu}\right)+m n \varphi\left(L_{-1}, M_{1-2 \mu}\right)=0, \forall n \in \mathbb{Z}, m \in \mathbb{Z}^{*}$ and further forces $\varphi\left(Y_{-\mu}, Y_{-\mu}\right)=\varphi\left(L_{-1}, M_{1-2 \mu}\right)=0$. Then recalling (2.52) and (2.60), we obtain

$$
\varphi\left(L_{-m-2 \mu}, M_{m}\right)=\varphi\left(Y_{-m-\mu}, Y_{m-\mu}\right)=0, \quad \forall m, n \in \mathbb{Z}
$$

If $\lambda=1$, using (2.53) and (2.57), we obtain

$$
\varphi\left(Y_{-m-\mu}, Y_{m-\mu}\right)=m\left(m^{2}-1\right) c_{1}-2 \varphi\left(Y_{-\mu}, Y_{-\mu}\right), \quad \forall m \in \mathbb{Z}^{*},
$$

for some constant $c_{1} \in \mathbb{C}$. Using (2.53), (2.62) and (2.56), we obtain $\varphi\left(Y_{-\mu}, Y_{-\mu}\right)=0$. Then (2.62) can be rewritten as

$$
\varphi\left(Y_{-m-\mu}, Y_{m-\mu}\right)=m\left(m^{2}-1\right) c_{1}^{\prime}, \quad \forall m \in \mathbb{Z}^{*}
$$

If $\lambda \notin\{-1,0,1\}$, taking $n=0$ in using (2.55) and (2.57), we obtain

$$
m(1+\lambda) \varphi\left(Y_{-m-\mu}, Y_{m-\mu}\right)=-m(3+\lambda) \varphi\left(Y_{-\mu}, Y_{-\mu}\right),
$$


which gives

$$
\varphi\left(Y_{-m-\mu}, Y_{m-\mu}\right)=-\frac{3+\lambda}{1+\lambda} \varphi\left(Y_{-\mu}, Y_{-\mu}\right), \quad \forall m \in \mathbb{Z}^{*}
$$

Taking (2.64) back to (2.56) $)$, one has $\varphi\left(Y_{-\mu}, Y_{-\mu}\right)=0$, which gives

$$
\varphi\left(Y_{-m-\mu}, Y_{m-\mu}\right)=0, \quad \forall m \in \mathbb{Z}
$$

Then this lemma follows.

Then the lemma proves Theorem 1.1 (ii)-(v).

Case 3. $\mu \in \mathbb{Z}$.

Lemma 2.3. (i) For the subcase $\lambda \neq-1$, one has $\varphi=0$.

(ii) For the subcase $\lambda=-1$, only $\varphi\left(Y_{-p}, Y_{p-2 \mu}\right)\left(\forall p \in \frac{1}{2}+\mathbb{Z}\right)$ is not vanishing, given in (2.71).

Proof. One has

$$
\begin{aligned}
& \varphi\left(L_{0}, Y_{p}\right)=0, \quad \forall p \in \frac{1}{2}+\mathbb{Z} \\
& \varphi\left(L_{0}, M_{n}\right)=0 \text { if } n \neq-2 \mu, \quad \varphi\left(L_{1}, M_{-2 \mu-1}\right)=0 \text { if } \lambda \neq-1 .
\end{aligned}
$$

For any $m, n \in \mathbb{Z}, p, q \in \frac{1}{2}+\mathbb{Z}$, according to the identities $(2.15)-(2.19),(2.66)$ and (2.67), one has

$$
\begin{aligned}
& (p+q+2 \mu) \varphi\left(Y_{p}, Y_{q}\right)=(m+n+2 \mu) \varphi\left(L_{m}, M_{n}\right)=0, \\
& \varphi\left(L_{0}, M_{-2 \mu}\right)=(m+n+4 \mu) \varphi\left(M_{m}, M_{n}\right)=\varphi\left(Y_{p}, M_{m}\right)=\varphi\left(L_{m}, Y_{p}\right)=0 .
\end{aligned}
$$

Similarly, one also can prove (2.20) holds in this case. Then from (2.66) $-(2.69)$, the left components we have to present in this case are listed in the following (where $m$ is an arbitrary integer):

$$
\varphi\left(Y_{-2 \mu-m}, Y_{m}\right) \text { and } \varphi\left(L_{-2 \mu-m}, M_{m}\right)
$$

which will be taken into account together in the following.

As in Case 2, the results (2.52)-(2.55) still hold in this case. Hence we shall cite them directly here using the same notations. Using the Jacobian identity on $\left(L_{-m}, Y_{-p}, Y_{m+p-2 \mu}\right)$, $\forall m \in \mathbb{Z}, p \in \frac{1}{2}+\mathbb{Z}$, one has

$$
\begin{aligned}
& (2 \mu-2 p+m(1+\lambda)) \varphi\left(Y_{-m-p}, Y_{m+p-2 \mu}\right) \\
& =2(m+2 p-2 \mu) \varphi\left(L_{-m}, M_{m-2 \mu}\right)-(2 p-2 \mu+m(3+\lambda)) \varphi\left(Y_{-p}, Y_{p-2 \mu}\right) .
\end{aligned}
$$


If $\lambda=-1$, then (2.70) becomes (recalling( $(2.54)$ )

$$
(\mu-p) \varphi\left(Y_{-m-p}, Y_{m+p-2 \mu}\right)+(p-\mu+m) \varphi\left(Y_{-p}, Y_{p-2 \mu}\right)=0,
$$

from which we can deduce

$$
\varphi\left(Y_{-p}, Y_{p-2 \mu}\right)=(p-\mu) c, \quad \forall p \in \frac{1}{2}+\mathbb{Z}
$$

for some constant $c \in \mathbb{C}$.

If $\lambda=0$, using (2.52) and (2.70), we obtain (taking $m=-1$ )

$$
(2(p+1)-(2 \mu+1)) \varphi\left(Y_{1-p}, Y_{p-1-2 \mu}\right)=(2(p-1)-(2 \mu+1)) \varphi\left(Y_{-p}, Y_{p-2 \mu}\right) .
$$

Using induction on $n=p-\frac{1}{2}$ where $p$ is that determined in (2.72), one has

$$
\varphi\left(Y_{-p}, Y_{p-2 \mu}\right)=(2(p+1)-(2 \mu+1))(2 p-(2 \mu+1)) c^{\prime},
$$

for some constant $c^{\prime} \in \mathbb{C}$. Taking $\varphi\left(L_{-m-2 \mu}, M_{m}\right)$ and $\varphi\left(Y_{-p}, Y_{p-2 \mu}\right)$ respectively given in (2.52) and (2.73) back to the identity (2.70), we obtain

$$
m(m+1)\left(4(m-1) \varphi\left(L_{-1}, M_{1-2 \mu}\right)+(2 p+2 \mu-m) c^{\prime}\right)=0, \quad \forall m \in \mathbb{Z}, p \in \frac{1}{2}+\mathbb{Z},
$$

which forces $\varphi\left(L_{-1}, M_{1-2 \mu}\right)=c^{\prime}=0$. Then (2.52) and (2.73) becomes

$$
\varphi\left(L_{-m-2 \mu}, M_{m}\right)=\varphi\left(Y_{-p}, Y_{p-2 \mu}\right)=0, \quad \forall m \in \mathbb{Z}, p \in \frac{1}{2}+\mathbb{Z} .
$$

If $\lambda=1$, using (2.53) and (2.70), we obtain (taking $m=-1$ )

$$
(p+1-\mu) \varphi\left(Y_{1-p}, Y_{p-1-2 \mu}\right)=(p-2-\mu) \varphi\left(Y_{-p}, Y_{p-2 \mu}\right) .
$$

Using induction on $n=p-\frac{1}{2}$ where $p$ is that given in (2.75), one has

$$
\varphi\left(Y_{-p}, Y_{p-2 \mu}\right)=(p-1-\mu)(p-\mu)(p+1-\mu) c^{\prime \prime}, \quad \forall p \in \frac{1}{2}+\mathbb{Z} .
$$

Taking $\varphi\left(L_{-m-2 \mu}, M_{m}\right)$ and $\varphi\left(Y_{-p}, Y_{p-2 \mu}\right)$ respectively given in (2.53) and (2.76) back to (2.70), one can deduce

$$
c_{1}^{\prime} m\left(m^{2}-1\right)(m+2 p-2 \mu)-c^{\prime \prime} m\left(m^{2}+p^{2}+m(p-\mu-1)-2 p(1+\mu)+\mu(2+\mu)\right),
$$

for any $m \in \mathbb{Z}, p \in \frac{1}{2}+\mathbb{Z}$, which forces $c_{1}^{\prime}=c^{\prime \prime}=0$. Then

$$
\varphi\left(L_{-m-2 \mu}, M_{m}\right)=\varphi\left(Y_{-p}, Y_{p-2 \mu}\right)=0, \quad \forall m \in \mathbb{Z}, p \in \frac{1}{2}+\mathbb{Z} .
$$

If $\lambda \notin\{-1,0,1\}$, using (2.55), one can rewrite (2.70) as (taking $m=1$ )

$$
(2 p-2 \mu-1-\lambda) \varphi\left(Y_{-1-p}, Y_{p+1-2 \mu}\right)=(2 p-2 \mu+3+\lambda) \varphi\left(Y_{-p}, Y_{p-2 \mu}\right) .
$$

Similarly, one also can deduce (2.65) holds in this case. Then this lemma follows.

Then the lemma proves Theorem 1.1 (vi). 


\section{References}

[1] B. Bakalov, V.G. Kac, A.A. Voronov, "Cohomology of conformal algebras," Comm. Math. Phys. 200 (1999), 561-598.

[2] S. Gao, C. Jiang, Y. Pei, Structure of the extended Schrödinger-Virasoro Lie algebra, arXiv:0801.2058v1, (2008); Alg. Colloq., in press.

[3] M. Henkel, Schrödinger invariance and strongly anisotropic critical systems, J. Stat. Phys., 75 (1994), 1023-1029.

[4] M. Henkel, Phenomenology of local scale invariance: from conformal invariance to dynamical scaling, Nucl. Phys. B, 641 (2002), 405-410.

[5] M. Henkel, J. Unterberger, Schrödinger invariance and space-time symmetries, Nucl. Phys. B, 660 (2003), 407-412.

[6] J. Li, Y. Su, The derivation algebra and automorphism group of the twisted SchrödingerVirasoro algebra, arXiv:0801.2207v1, (2008).

[7] J. Li, Y. Su, Representations of the Schrödinger-Virasoro algebras, arXiv:0801.2209v1, (2008).

[8] J. Li, Y. Su, 2-Cocycles of Twisted Deformative Schrödinger-Virasoro Algebras, arXiv: 0801.2210v1, (2008).

[9] W. Li, "2-Cocycles on the algebra of differential operators," J. Alg. 122 (1989), 64-80.

[10] W. Li, R.L. Wilson, Central extensions of some Lie algebras, Proc. Amer. Math. Soc. 126 (1998), 2569-2577.

[11] C. Roger, J. Unterberger, The Schrödinger-Virasoro Lie group and algebra: from geometry to representation theory, arXiv:math-ph/0601050v1, (2006).

[12] M. Scheunert, R.B. Zhang, "Cohomology of Lie superalgebras and their generalizations," J. Math. Phys. 39 (1998), 5024-5061.

[13] Y. Su, "2-Cocycles on the Lie algebras of all differential operators of several indeterminates," (Chinese) Northeastern Math. J. 6 (1990), 365-368.

[14] Y. Su, "2-cocycles on the Lie algebras of generalized differential operators", Comm. Algebra 30 (2002), 763-782.

[15] Y. Su, "Low dimensional cohomology of general conformal algebras $g c_{N}$," J. Math. Phys. 45 (2004), 509-524.

[16] Y. Su, K. Zhao, "Second cohomology group of generalized Witt type Lie algebras and certain reperesentations," Comm. Alegrba 30 (2002), 3285-3309.

[17] J. Unterberger, On vertex algebra representations of the Schrödinger-Virasoro algebra, arXiv:cond-mat/0703214v2, (2007). 